\title{
Brain Mapping of Patients with Lung Cancer and Controls: Inquiry into Tumor-to-Brain Communication
}

\author{
Haim Golan ${ }^{1}$, John A. Kennedy ${ }^{2}$, Alex Frenkel ${ }^{2}$, Ysrael Parmet ${ }^{3}$, Akiva Feintuch ${ }^{3}$, Ofer Levi ${ }^{3}$, and Yori Gidron ${ }^{4}$ \\ ${ }^{1}$ Department of Nuclear Medicine, Assaf Harofeh Medical Center, Zerifin, Israel; ${ }^{2}$ Department of Nuclear Medicine, \\ Rambam Health Care Campus, Haifa, Israel; ${ }^{3}$ Department of Industrial Engineering and Management, Ben-Gurion University, \\ Beer-Sheva, Israel; and ${ }^{4}$ School of Health Sciences and Social Care, Brunel University, West London, United Kingdom
}

\begin{abstract}
Recent converging evidence suggests that the brain may receive stimuli and possibly modulate tumor progression via the vagus nerve. The present study aimed to compare brain metabolism in patients with and without lung cancer and to assess if significant differences exist in regions associated with the vagus nerve. Methods: Eighteen patients with lung malignancy and 19 controls underwent ${ }^{18} \mathrm{~F}-\mathrm{FDG}$ PET of the brain. Brain metabolism was compared using statistical parametric mapping. Results: Patients with lung malignancy showed a statistically significantly higher right cerebellar metabolism. Conclusion: This finding may be related to the role of the cerebellum in immune regulation, because of its proximity to the nucleus tractus solitarius innervated by the vagus and its connections with the hypothalamus. This higher metabolism in the right cerebellum may reflect an attempt to reinstate homeostasis in functions such as respiration and immunity pertinent to lung malignancy.
\end{abstract}

Key Words: lung cancer; vagus nerve; FDG brain PET; tumorto-brain communication

J Nucl Med 2009; 50:1072-1075

DOI: 10.2967/jnumed.108.061085

\footnotetext{
C
} ancer research has shifted from solely focusing on genetic aspects and defining the intracellular etiology of tumorigenesis to also assessing interactions between tumors and their microenvironment (1). Furthermore, there is increasing interest in assessing the potential presence of bidirectional links between peripheral malignant tumors and the brain, in the form of tumor-to-brain communication and modulation (2).

The inflammatory microenvironment plays a crucial role early during tumorigenesis $(3,4)$ and later for angiogenesis and tumor invasion (5). The brain receives signals from peripheral inflammatory processes by several routes, including the vagus $(6)$, through interleukin-1 receptors on its paraganglia. There are conflicting reports on the rate of

Received Dec. 25, 2008; revision accepted Apr. 24, 2009.

For correspondence or reprints contact: Haim Golan, Department of

Nuclear Medicine, Assaf Harofeh Medical Center, Zerifin 70300, Israel. E-mail: golanh@asaf.health.gov.il

COPYRIGHT @ 2009 by the Society of Nuclear Medicine, Inc. cancer-related death in vagotomized ulcer patients $(7,8)$. However, chemical and surgical vagotomy prevents tumorassociated anorexia in mice (9) and enhances the metastasizing rate of peripheral malignant tumors (10). In view of these studies, it has been hypothesized that the vagus (and potentially other neural routes) may provide signals about the presence of malignancy-related inflammation to the brain with possible subsequent modulation of tumorigenesis $(2,11)$.

The present study aimed to examine brain metabolism in patients with lung malignancy, addressing most of the limitations of prior studies (12-15). Most patients included in the study were evaluated because of pulmonary nodules detected on CT. Both patients and physicians were unaware of the final diagnosis with respect to the presence of active malignancy at the time of imaging, which was performed before any treatment.

\section{MATERIALS AND METHODS}

\section{Patients and Control Subjects}

Eighteen patients with lung malignancy and 19 controls were included. The malignancy group consisted only of newly diagnosed, histologically proven cases of cancer and included 16 patients with non-small cell lung cancer (stage 1A or $1 \mathrm{~B}[n=4]$, $2 \mathrm{~A}$ or $2 \mathrm{~B}[n=3], 3 \mathrm{~A}$ or $3 \mathrm{~B}[n=4]$, or $4[n=5]), 1$ patient with extensive small cell lung cancer, and 1 patient with diffuse large cell non-Hodgkin lymphoma of the lung. The mean size of the lung lesions was $38.6 \pm 19.5 \mathrm{~mm}$, and the range was $10-80 \mathrm{~mm}$. Nine lesions were in the right lung (upper lobe $[n=1]$, middle lobe $[n=1]$, or lower lobe $[n=7])$, and 9 were in the left lung (upper lobe $[n=5]$, lingula $[n=1]$, or lower lobe $[n=3]$ ).

The control group comprised a subgroup of 8 patients with lymphoma in remission for at least $4 \mathrm{y}$ (the LymC group), including Hodgkin lymphoma $(n=2$, supraclavicular nodes at presentation), diffuse large cell non-Hodgkin lymphoma $(n=2$, inguinal nodes in the first patient and supraclavicular and mediastinal nodes in the second patient at presentation), follicular nonHodgkin lymphoma $(n=2$, inguinal nodes in both patients at presentation), and primary bone non-Hodgkin lymphoma $(n=2$, multiple skeletal lesions in both patients at presentation). The patients had been in continuous clinical remission for a mean of 
$76.3 \pm 27.7 \mathrm{mo}$ (range, $54-132 \mathrm{mo}$ ) at the time of the routine follow-up ${ }^{18}$ F-FDG PET/CT study and showed no further evidence of recurrence.

The second control subgroup comprised 11 patients with benign thoracic lesions including 8 lung nodules and 3 mildly enlarged mediastinal lymph nodes in which histology or long-term clinical follow up did not show any evidence of malignancy. The mean size of the 8 lung nodules was $14.8 \pm 5.2 \mathrm{~mm}$, and the range was $8-20 \mathrm{~mm}$. One nodule was in the right lower lobe. Seven were in the left lung, and 5 of these 7 involved mainly the upper lobe. Histologic examinations had been performed on 5 patients; the results were negative in 3 and indicated fibrotic changes and hamartoma in 1 patient each. The 3 other patients had no evidence of malignancy at a clinical follow-up of 13, 31, and 36 mo. The 3 enlarged mediastinal lymph nodes were 10,10 , and $15 \mathrm{~mm}$ in diameter. Biopsy was negative in 2 patients and found lipoma in 1 patient.

Exclusion criteria included brain tumors, mental illness, prior electroconvulsive therapy, organic brain disorders, head trauma, a major medical illness (e.g., hypo- or hyperthyroidism, vitamin B12 deficiency, diabetes, renal failure, hepatic failure, chronic heart disease, and autoimmune diseases), a history of drug or alcohol dependence, or abdominal and visceral infections to rule out non-tumor-related effects on the brain.

The patients with malignancy (15 men and 3 women) had a mean age of $66.9 \pm 9.0 \mathrm{y}$ and an age range of $43-80 \mathrm{y}$. The mean age of all control patients (12 men and 7 women) was $64.2 \pm 9.8 \mathrm{y}$, and the age range was 50-78 y. Patients in the LymC group had a mean age of $61.3 \pm 8.6 \mathrm{y}$ and an age range of 50-72 y, whereas those in the no-malignancy group had a mean age of $66.0 \pm 10.1 \mathrm{y}$ and an age range of 50-78 y.

\section{${ }^{18}$ F-FDG PET of the Brain}

Data were acquired using a clinical PET/CT scanner (Discovery LS; GE Healthcare). Participants fasted for at least $4 \mathrm{~h}$ before being injected with $370-555 \mathrm{MBq}(10-15 \mathrm{mCi})$ of ${ }^{18} \mathrm{~F}-\mathrm{FDG}$ and then rested for $60 \mathrm{~min}$ in a quiet room, with dimmed light and eyes opened. They were instructed to refrain from reading, listening to music, and talking during the uptake period. The brain scan consisted of a 1-field-of-view CT scan followed by a 15-min PET study performed at $60 \mathrm{~min}$ after ${ }^{18}$ F-FDG injection. Subsequently, a separate whole-body PET/CT study was performed for diagnosis at 90 min after injection. Transaxial 2-dimensional PET data from the brain scan (35 slices) were reconstructed using an orderedsubsets expectation maximization algorithm (2 iterations, 28 subsets) as $128 \times 128$ pixel images having a pixel size of $2.3 \times$ $2.3 \mathrm{~mm}$ and a slice thickness of $4.25 \mathrm{~mm}$. These were smoothed with a gaussian filter of $3 \mathrm{~mm}$ in full width at half maximum. The CT data were used for PET attenuation correction

\section{Data Analysis}

SPM-5 (Wellcome Department of Cognitive Neurology, Institute of Neurology, University College London) implanted in MATLAB 7 (The MathWorks) was used for image processing. PET images were interpolated to a $2 \times 2 \times 2 \mathrm{~mm}$ voxel size (trilinear interpolation), spatially normalized to the standard PET template embedded in SPM-5, adjusted to the Talairach stereotactic brain atlas, and further smoothed with a gaussian kernel of $10 \mathrm{~mm}$ in full width at half maximum. Global normalization and proportional scaling with 0.8 threshold masking were applied.

The 2-sample $t$ test, adjusted for sex to control for its potential confounding effects, was used for group comparisons between the controls with no malignancy or those with LymC, and patients with malignancy. We used $\mathrm{F}$ contrast, meaning that no directionality in the contrasts between the 2 control groups was assumed before analysis. After establishing that there were no significant differences between the no-malignancy and LymC groups, we merged them into a single control group $(n=19)$ and then compared that combined group with the malignancy group $(n=$ 18).

The controls and the malignancy patients were compared using the working hypothesis that increased ${ }^{18}$ F-FDG uptake will be found in the brain of the malignancy group since tumor-related inflammation was expected to lead to greater activity in the brain, in line with immune-to-brain communication. Therefore, T contrast was used. The extent threshold, k, was 10 voxels. Falsediscovery-rate correction for multiple comparisons, q, was 0.1 . The brain activity change between groups was considered significant if more than 4 voxels $\left(32 \mathrm{~mm}^{3}\right)$ were affected.

For each given set of SPM-5 Talairach space coordinates of brain maxima activity, a cube range search was performed using Talairach Client software (version 2.4.2; Talairach.org). A cube range search of $\pm 5 \mathrm{~mm}$ (11 mm wide) returned all Talairach atlas labels within the cube centered on the primary maxima activity $x, y, z$-coordinates generated by the SPM-5 analysis.

\section{RESULTS}

No significant age differences were found between subjects with no malignancy, LymC, and malignancy $(P=$ 0.363 ). No statistically significant difference in ${ }^{18} \mathrm{~F}-\mathrm{FDG}$ brain uptake was found between the 2 control subgroups.

SPM-5 analysis found increased ${ }^{18} \mathrm{~F}$-FDG brain uptake in the right cerebellum of the malignancy group (Fig. 1). The coordinates of the primary and secondary maxima are 20 , $-64,-24$ and $6,-64,-16$, respectively. Both points were in the marked area and were statistically significant (adjusted $P<0.01$ using a false-discovery rate of $\mathrm{q}=0.1$ ).

\section{DISCUSSION}

The present study aimed to compare brain metabolism between patients who had malignancy and controls who had no evidence of active malignancy, including patients with lymphoma in remission and patients who had been referred for PET/CT because of a newly diagnosed thoracic morphologic abnormality but had proven to be free of

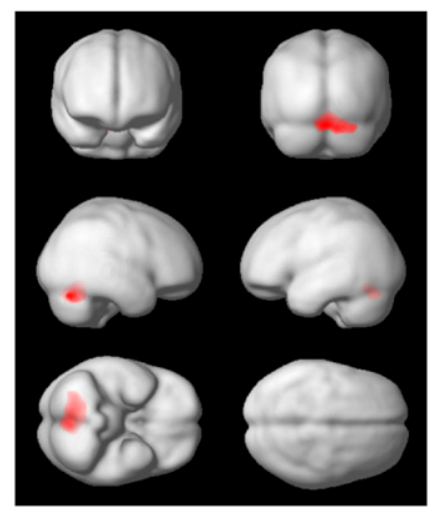

FIGURE 1. Right cerebellar activation region of patients with lung cancer $(n=18)$ vs. controls $(n=$ 19), depicted on SPM-5 standard brain template. This activated brain region was subjected to false-discovery-rate correction for multiple comparisons, which found significant difference of $P<0.01$ and with minimum of 10 voxels. 
cancer for at least $1 \mathrm{y}$ after the initial scan. Because preliminary analysis showed no significant differences between the 2 control subgroups, their data were merged and compared with those of the malignancy cases to increase our statistical power. The results demonstrated a higher brain metabolism in patients with malignancy than in controls, limited to the right cerebellum.

These findings are in contrast to those of Tashiro et al. $(12,13)$, who reported hypoactivity in the anterior and posterior cingulate gyri, the basolateral frontal cortex, and the caudate nucleus. This pattern was considered to represent a depressive state. The studies of Tashiro et al. were hampered by methodologic limitations-mainly a study group heterogeneity consisting of a variety of malignancies and neuroimaging assessments during or after a variety of therapies, which could also influence brain activity $(14,15)$. In addition, the fact that the patients were aware of their diagnoses may, at least in part, explain the observed depressive brain activity pattern. In the present study, patients were evaluated without knowledge of their final diagnosis and before receiving any cancer therapy.

How can the significantly increased right cerebellar metabolism in patients with malignancy be related to the vagal nerve hypothesis of tumor modulation? Turrin et al. (16) have reported that intraperitoneal administration of bacterial lipopolysaccharide induced an increase in interleukin- $1 b$ and tumor necrosis factor- $\alpha$ messenger RNA in several brain regions, including the cerebellum. Thus, because this region responds to peripheral inflammation, it may also respond to tumor-associated inflammation. A recent study has described a relationship between vagotomy and breast cancer metastases to the lungs and other regions (10). The increase in substance $\mathrm{P}$ associated with the presence of a malignant tumor and with vagotomy may reflect an increase in tumor-associated inflammation, with inflammation and pain being strongly linked. This study further supports the present results regarding a potential role of the vagus in tumor-to-brain communication and modulation.

Cerebellar symptoms have also been reported in paraneoplastic syndromes and in other mimicking conditions (17). However, it seems statistically improbable that all our patients with lung malignancies had this rare neurologic disorder.

The cerebellum plays an important role in memory, emotion, feeding, micturition, immune response, and respiratory and cardiovascular functions (18). Afferent connections from the cerebellum to the posterior and dorsomedial nuclei of the hypothalamus explain, in part, the regulatory effects of the cerebellum on visceromotor functions (19). The role of the cerebellum in immune regulation (possibly significant in tumor modulation), its proximity to the nucleus tractus solitarii innervated by the vagus, and its above-mentioned connections to the hypothalamus may explain at least in part the increased metabolism we found in patients with malignancy, compared with controls. These all may reflect an attempt of the body to regain homeostasis.
This study had several limitations. A healthy control group would have been a good additional reference group but would have been difficult to justify. The ideal study populations would consist only of patients scanned for the first time (unlike the LymC group), since anxiety-related arousal might be less prominent in patients who have already been scanned multiple times. Although lymphoma patients had received treatment in the past, potential effects of previously administered chemotherapy in the LymC group were not considered. However, any effects were expected to be minimal because brain uptake of ${ }^{18} \mathrm{~F}-\mathrm{FDG}$ did not significantly differ between the 2 control subgroups. Finally, because the study lacked psychologic measures, the effects of psychologic factors or other confounding factors could not be tested.

These preliminary results suggesting the presence of hypermetabolism in the cerebellum in patients with malignancy need to be confirmed by further studies that include large numbers of patients. Future studies also need to investigate why the right cerebellum, and not the left or both cerebella, was hyperactive in the malignancy cases, given known lateralization and immunity findings (20). Finally, the prognostic role of these findings should also be investigated in the future.

\section{CONCLUSION}

In this study, patients with lung malignancy showed a statistically significantly higher right cerebellar metabolism. This finding may be related to the role of the cerebellum in immune regulation because of its proximity to the nucleus tractus solitarius innervated by the vagus and its connections with the hypothalamus. This higher metabolism in the right cerebellum may reflect an attempt to reinstate homeostasis in functions such as respiration and immunity pertinent to lung malignancy.

\section{ACKNOWLEDGMENT}

We are deeply grateful to Prof. Ora Israel of Rambam Health Care Campus, Haifa, Israel, for her help, effort, and intellectual contribution to this work.

\section{REFERENCES}

1. Mbeunkui F, Johann DJ Jr. Cancer and the tumor microenvironment: a review of an essential relationship. Cancer Chemother Pharmacol. 2009;63:571-582.

2. Gidron Y, Perry H, Glennie M. Does the vagus nerve inform the brain about preclinical tumours and modulate them? Lancet Oncol. 2005;6:245-248.

3. Pikarsky E, Porat RM, Stein I, et al. NF-kappa B functions as a tumour promoter in inflammation-associated cancer. Nature. 2004;431:461-466.

4. Greten FR, Eckmann L, Greten TF, et al. IKKbeta links inflammation and tumorigenesis in a mouse model of colitis-associated cancer. Cell. 2004; 118:285-296.

5. Voronov E, Shouval DS, Krelin Y, et al. IL-1 is required for tumor invasiveness and angiogenesis. Proc Natl Acad Sci USA. 2003;100:2645-2650.

6. Ek M, Kurosawa M, Lundeberg T, Ericsson A. Activation of vagal afferents after intravenous injection of interleukin-1beta: role of endogenous prostaglandins. J Neurosci. 1998;18:9471-9479.

7. Ekbom A, Lundegårdh G, McLaughlin JK, Nyrén O. Relation of vagotomy to subsequent risk of lung cancer: population based cohort study. BMJ. 1998; 316:518-519. 
8. Lundegårdh G, Ekbom A, McLaughlin JK, Nyrén O. Gastric cancer risk after vagotomy. Gut. 1994;35:946-949.

9. Bernstein IL. Neutral mediation of food aversions and anorexia induced by tumor necrosis factor and tumors. Neurosci Biobehav Rev. 1996;20: $177-181$.

10. Erin N, Barkan GA, Harms JF, Clawson GA. Vagotomy enhances experimental metastases of 4THMpc breast cancer cells and alters Substance P level. Regul Pept. 2008;151:35-42.

11. Mravec B, Gidron Y, Kukanova B, Bizik J, Kiss A, Hulin I. Neural-endocrineimmune complex in the central modulation of tumorigenesis: facts, assumptions, and hypotheses. J Neuroimmunol. 2006;180:104-116.

12. Tashiro M, Itoh M, Kubota K, et al. Relationship between trait anxiety, brain activity and natural killer cell activity in cancer patients: a preliminary PET study. Psychooncology. 2001;10:541-546.

13. Tashiro M, Juengling FD, Reinhardt MJ, et al. Reproducibility of PET brain mapping of cancer patients. Psychooncology. 2000;9:157-163.

14. Matsuda T, Takayama T, Tashiro M, Nakamura Y, Ohashi Y, Shimozuma K. Mild cognitive impairment after adjuvant chemotherapy in breast cancer patients: evaluation of appropriate research design and methodology to measure symptoms. Breast Cancer. 2005;12:279-287.

15. Silverman DHS, Dy CJ, Castellon SA, et al. Altered frontocortical, cerebellar, and basal ganglia activity in adjuvant-treated breast cancer survivors 5-10 years after chemotherapy. Breast Cancer Res Treat. 2007;103:303-311.

16. Turrin NP, Gayle D, Ilyin SE, et al. Pro-inflammatory and anti-inflammatory cytokine mRNA induction in the periphery and brain following intraperitoneal administration of bacterial lipopolysaccharide. Brain Res Bull. 2001;54:443-453.

17. Darnell RB. Paraneoplastic syndromes. In: Current Diagnosis in Neurology. Feldmann E, ed. St. Louis, MO: Mosby-Year Book, Inc.; 1994:137-141.

18. Zhu JN, Yung WH, Kwok-Chong Chow B, Chan YS, Wang JJ. The cerebellarhypothalamic circuits: potential pathways underlying cerebellar involvement in somatic-visceral integration. Brain Res Rev. 2006;52:93-106.

19. Onat F, Cavdar S. Cerebellar connections: hypothalamus. Cerebellum. 2003;2:263-269.

20. Meador KJ, Loring DW, Ray PG, Helman SW, Vazquez BR, Neveu PJ. Role of cerebral lateralization in control of immune processes in humans. Ann Neurol. 2004;55:840-844. 